\title{
Article
}

\section{New Promoters for Metabolic Engineering of Ashbya gossypii}

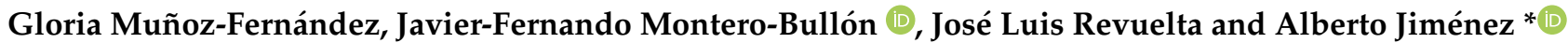

Metabolic Engineering Group, Department of Microbiology and Genetics, University of Salamanca, 37007 Salamanca, Spain; gmf@usal.es (G.M.-F.); jfmonbul@usal.es (J.-F.M.-B.); revuelta@usal.es (J.L.R.)

* Correspondence: alji@usal.es; Tel.: +34-677-527-004

check for updates

Citation: Muñoz-Fernández, G.; Montero-Bullón, J.-F.; Revuelta, J.L.; Jiménez, A. New Promoters for Metabolic Engineering of Ashbya gossypii. J. Fungi 2021, 7, 906. https:// doi.org/10.3390/jof7110906

Academic Editor:

Ivan-Kresimir Svetec

Received: 5 October 2021

Accepted: 24 October 2021

Published: 26 October 2021

Publisher's Note: MDPI stays neutral with regard to jurisdictional claims in published maps and institutional affiliations.

Copyright: (c) 2021 by the authors. Licensee MDPI, Basel, Switzerland. This article is an open access article distributed under the terms and conditions of the Creative Commons Attribution (CC BY) license (https:// creativecommons.org/licenses/by/ $4.0 /)$.

\begin{abstract}
Ashbya gossypii is a filamentous fungus that is currently exploited for the industrial production of riboflavin. In addition, metabolically engineered strains of $A$. gossypii have also been described as valuable biocatalysts for the production of different metabolites such as folic acid, nucleosides, and biolipids. Hence, bioproduction in A. gossypii relies on the availability of wellperforming gene expression systems both for endogenous and heterologous genes. In this regard, the identification of novel promoters, which are critical elements for gene expression, decisively helps to expand the A. gossypii molecular toolbox. In this work, we present an adaptation of the Dual Luciferase Reporter (DLR) Assay for promoter analysis in A. gossypii using integrative cassettes. We demonstrate the efficiency of the analysis through the identification of 10 new promoters with different features, including carbon source-regulatable abilities, that will highly improve the gene expression platforms used in $A$. gossypii. Three novel strong promoters $\left(P_{C C W 12}, P_{S E D 1}\right.$, and $\left.P_{\text {TSA1 }}\right)$ and seven medium/weak promoters $\left(P_{H S P 26}, P_{A G L 366 C}, P_{T M A 10}, P_{C W P 1}, P_{A F R 038 \mathrm{~W}}, P_{P F S 1}\right.$, and $\left.P_{C D A 2}\right)$ are presented. The functionality of the promoters was further evaluated both for the overexpression and for the underexpression of the A. gossypii MSN2 gene, which induced significant changes in the sporulation ability of the mutant strains.
\end{abstract}

Keywords: Ashbya gossypii; gene expression; promoter; luciferase; metabolic engineering

\section{Introduction}

Ashbya gossypii is a filamentous hemiascomycete of the Saccharomycetaceae family with industrial relevance since it is a natural overproducer of riboflavin (vitamin B2). Indeed, engineered strains of $A$. gossypii are currently exploited as microbial factories for the industrial production of riboflavin [1,2]. Besides the utilization of A. gossypii for riboflavin production, during the last decade, this fungus has emerged as a potential microbial factory for the production of other purine-related metabolites such as nucleosides and folic acid [3,4], and other bioproducts with industrial interest such as proteins, biolipids, and gamma-lactones [5-7]. In addition, industrial residues and by-products such as lignocellulosic hydrolysates, molasses, and crude glycerol have been documented as efficient carbon sources for A. gossypii [8,9].

The importance of $A$. gossypii in industry owes itself to certain beneficial aspects regarding bioprocessing and metabolic engineering. First, the bioprocessing is cost-effective since $A$. gossypii has the ability to grow using low-cost substrates and the downstream processing is inexpensive [10]. Second, there is a large and efficient molecular toolbox for the genomic manipulation of the fungus, including gene-targeting methods, heterologous expression platforms, or CRISPR/Cas9/Cas12 adapted systems [6,11-13].

Systems metabolic engineering applied to produce complex, high added-value compounds requires well-performing gene expression tools. In S. cerevisiae, the production of the antimalarial artemisinic acid required seven engineering steps to deregulate the mevalonate pathway and redirect metabolic flux towards artemisinic acid [14]. In Yarrowia lipolytica, 30 copies of nine different genes were used to produce high levels of the omega-3 fatty acid eicosapentaenoic acid [15]. More recently, the yeast Pichia pastoris has been 
engineered to grow on $\mathrm{CO}_{2}$ using up to eight heterologous genes [16]. All those successful approaches are based on the availability of a large collection of constitutive and inducible promoters that enable the use of several different expression platforms.

The manipulation of $A$. gossypii is restricted to genomic integrations since no stable plasmids exist for this fungus. Hence, gene deletions, as well as gene underexpression and overexpression, are carried out using integrative cassettes [17,18]. Likewise, heterologous gene expression is accomplished by using single/multiple overexpression platforms with a single integrative cassette [13]. The utilization of those molecular tools in A. gossypii enabled the simultaneous modification/engineering of up to eight genes for the production of polyunsaturated fatty acids from glucose [13] and microbial oils from xylose-based residues [8]. However, the recurrent utilization of the same strong promoter for gene overexpression can affect the fitness of the engineered strains, probably due to the presence of several identical sequences, and, thereby, limit the number of manipulations to be introduced in the genome. In this regard, to develop optimized expression platforms in $A$. gossypii, the identification of native promoters covering different expression patterns will highly improve the molecular toolbox for this fungus.

While several strong native constitutive promoters are used in this fungus such as $P_{G P D 1}, P_{T E F}, P_{P G K 1}$, and $P_{A D H 1}[6,13]$, only one weak native promoter from $A g R I B 7$ has been reported for gene underexpression [17]. Additionally, a few regulatable promoters have been described in A. gossypii, such as $P_{M E T 3}$ [19], for methionine-dependent downregulation, or the thiamine-repressible S. cerevisiae THI13 promoter [20].

In this context, plasmid-based GFP- and lacZ-reporter assays were previously described for promoter analysis in A. gossypii [19]. However, as mentioned above, plasmids are not fully stable in the multinucleated syncytium of $A$. gossypii, and, moreover, the variability in the plasmid copy number can add experimental inaccuracy. Hence, the development of new methods for promoter analysis in A. gossypii using integrative cassettes is desirable.

In this work we present a method for promoter analysis in A. gossypii using integrative cassettes based on the mammalian Dual Luciferase Reporter (DLR) Assay, which allows the sequential quantitative measurement of two luciferase activities (Renilla and firefly luciferases) in a single protein extract, thus conferring accuracy and reproducibility to the system. We report 10 new promoters covering a wide range of promoter activity with important potential applications for metabolic engineering of A. gossypii.

\section{Materials and Methods}

\subsection{Ashbya gossypii Strains and Growth Conditions}

The A. gossypii ATCC 10,895 strain was used as the wild-type strain. The A. gossypii strains generated in this study are listed in Table S1. A. gossypii liquid cultures were initiated with spores $\left(10^{6}\right.$ spores/L) and carried out at $28^{\circ} \mathrm{C}$ and $200 \mathrm{rpm}$ using MA2-rich medium ( $2 \%$ bactopeptone, $0.2 \%$ yeast extract, $0.06 \%$ myo-inositol, and $\mathrm{pH} 6.8$ ) with the indicated carbon source, either $2 \%$ glucose or $2 \%$ oleic acid (OA) plus $0.5 \%$ glucose. A. gossypii transformation, spore isolation, and sporulation conditions were as described in [21]. A concentration of $250 \mathrm{mg} / \mathrm{L}$ for geneticin (G418) (Gibco-BRL, Waltham, MA, USA) was used where indicated.

\subsection{Assembly and Genomic Integration of the Cassettes for Renilla and Firefly Luciferase Expression}

The integrative cassettes used in this work were assembled using a Golden Gate method, as described in [13]. The integrative cassettes comprised recombinogenic flanks targeting either the ADR304W or AGL034C loci, a loxP-KanMX-loxP $\left(\mathrm{G} 418^{\mathrm{R}}\right)$ marker, and the transcriptional units for either Renilla or firefly luciferase expression (Table S2-NCBI accession numbers: Renilla luciferase, AAB82577.1; firefly luciferase, AAA29795.1). For the expression of the Renilla luciferase, the strong constitutive promoter $P_{G D P 1}$ was used; for the expression of the firefly luciferase, different promoter sequences were used. The 
CDS of Renilla and firefly luciferases were PCR-amplified from the pRL-SV40 (Promega; NCBI accession number: AF025845.2) and pAP1-luc (Stratagene; NCBI accession number: AF053698.1), respectively. In addition, the intergenic promoter sequences were PCRamplified from A. gossypii genomic DNA. Those PCR primers contained a BsaI recognition site and 4-nucleotide overhangs for the Golden Gate assembly (see Table S3 for primer sequences). All the modules were assembled into a destination vector that contains a spectinomycin resistance marker (Figure S1). The assembled plasmids were selected in spectinomycin/kanamycin-containing LB plates and were confirmed by restriction analysis and DNA sequencing. The final integrative cassettes were isolated by SapI digestion and used for A. gossypii transformation.

Spores of A. gossypii were transformed with the integrative cassettes, and positive primary heterokaryotic clones were selected in G418-containing medium. Homokaryotic clones were obtained by sporulation of the primary transformants. The correct genomic integration of each integrative cassette was confirmed by analytical PCR followed by DNA sequencing. The transient expression of a Cre recombinase enabled the loxP-kanMX-loxP marker to be eliminated and reused, as described elsewhere [22].

\subsection{Dual Luciferase Reporter (DLR) Assay for Promoter Analysis}

A DLR assay system (Promega, Madison, WI, USA) was used for promoter analysis. Flask cultures for luciferase assays were initiated with $10^{5}$ spores and carried out in $30 \mathrm{~mL}$ of MA2 rich medium at $28{ }^{\circ} \mathrm{C}$ for $40 \mathrm{~h}$. A mycelial biomass of $100 \mu \mathrm{L}$ was harvested, washed twice with $200 \mu \mathrm{L}$ of PBS and resuspended in $100 \mu \mathrm{L}$ of Passive lysis Buffer from the DLR assay kit (Promega). Cell disruption was carried out with glass beads $(0.5 \mathrm{~mm})$ by vortexing 4 times for $15 \mathrm{~s}$, each time keeping the cells on ice for $1 \mathrm{~min}$ between vortexing. The luciferase assay was performed following the manufacturer's instructions of the DLR assay kit (Promega). Luminescence of both Renilla and firefly luciferases were measured sequentially using a Varioskan microtiter plate reader (Thermo Scientific, Waltham, MA, USA). The results were represented as a ratio of the luciferase activity of firefly and Renilla (ratio Fluc/Rluc), which allows for a normalization of the results with an internal control.

\subsection{Construction and Integration of MSN2 Expression Cassettes}

Different integrative cassettes targeting the AgMSN2 (ABR089C) gene were PCRamplified from the firefly expression cassettes described above using the primers listed in Table S3. Each cassette was integrated upstream of the ATG initiator codon of AgMSN2 by homologous recombination using recombinogenic flanks that were included in the primer sequences. The expression cassettes comprised a different promoter sequence and the loxP-KanMX-loxP $\left(\mathrm{G} 418^{\mathrm{R}}\right)$ selectable marker. Genomic integration of the expression cassettes was confirmed by analytical PCR.

\subsection{Quantitative Real-Time PCR}

Quantitative real-time PCR (qRT-PCR) was performed with a LightCycler 480 realtime PCR instrument (Roche, Basel, Switzerland), using SYBR Green I master mix (Roche) following the manufacturer's instructions. Total RNA samples were prepared as described in [23]. cDNA was synthesized using the Transcriptor First Strand cDNA Synthesis Kit (Roche). Primer sequences are listed in Table S3. qRT-PCR reactions were performed in duplicate and in at least two independent experiments. Quantitative analyses were carried out using the LightCycler 480 software. The mRNA level of the target genes was normalized to that of $A g U B C 6$ and was calculated using the $2^{-\Delta \Delta C t}$ method [24].

\subsection{RNAseq}

The A. gossypii WT strain was grown in $50 \mathrm{~mL}$ of MA2 flask cultures for $72 \mathrm{~h}$. Mycelia were harvested, and total RNA samples were prepared as described in [23]. Total RNA was used for Illumina Hiseq 2000 sequencing. RNAseq was performed by Macrogen (Seoul, South Korea). Raw data were processed, and reads were aligned to the A. gossypii reference 
genome using Geneious R11 software 10.0.5. The expression level for each coding sequence was calculated based on the normalized FPKM (fragments per kb/million mapped reads).

\subsection{Sporulation Analysis}

Mycelia of selected A. gossypii strains were cultured onto sporulation (SPA, sporulation of Ashbya) media plates for 4 days at $28^{\circ} \mathrm{C}$ and spores were isolated from $100 \mathrm{mg}$ of mycelia that were scraped out from each plate. Serial dilutions of the spore preparations were performed in $0.01 \%$ Triton X-100, plated on MA2 medium, and incubated at $28{ }^{\circ} \mathrm{C}$ until colonies appeared. Additionally, the number of spores from $10^{-2}$ dilutions were counted using a Neubauer cell chamber. Data are expressed as the number of spores/g of mycelium of each strain.

\section{Results and Discussion}

\subsection{Adaptation of a Luciferase Reporter Assay for Promoter Analysis in A. gossypii}

A luciferase reporter assay was designed to evaluate the ability of different promoter sequences to drive gene expression in A. gossypii. Since episomic vectors are not fully stable in A. gossypii, the promoter activities were assessed using genomic integrative cassettes. The coding sequences of the Renilla (Renilla reniformis) and firefly (Photinus pyralis) luciferases were used in a dual luciferase reporter assay to improve experimental accuracy. Hence, two integrative cassettes were assembled: an integrative module for the expression of the Renilla luciferase was used as the internal control, and an integrative cassette for the firefly luciferase was used as the experimental reporter. Each genomic integrative cassette comprised recombinogenic flanks, a loxP-KanMX-loxP $\left(\mathrm{G} 418^{\mathrm{R}}\right)$ marker, the promoter sequence, the reporter luciferase CDS, and the terminator sequence of $P G K 1$ (Figure 1). The recombinogenic flanks target the ADR304W and AGL034C loci in the integrative cassette for Renilla and firefly, respectively. The disruption of either $A D R 304 \mathrm{~W}$ or AGL034C loci does not affect growth in A. gossypii, as previously described in [25]. In this work, the strong promoter $P_{G P D 1}$ was chosen as the internal control. The integrative cassettes were assembled following a Golden Gate modular cloning system adapted for $A$. gossypii, (Figure 1) [13].

For the construction of the internal control strain, a wild-type strain of A. gossypii was first transformed with the integrative module for Renilla luciferase expression to generate strain A846 (Figure 2A). The G418 ${ }^{\mathrm{R}}$ marker was eliminated in the A846 strain by Cre recombinase expression to generate control strain A848. This strain was transformed with the corresponding cassette for firefly expression to obtain strain A855. The integration of the expression cassettes in the target loci was verified by analytical PCR (Figure 2B,C). After marker elimination, an initial strain, named A947, was obtained (Figure 2A), which was equipped with both the Renilla and firefly expression cassettes containing the strong promoter $P_{G P D 1}$. The expression of both Renilla and firefly luciferases from $P_{G P D 1}$ in strain A947 was analyzed using the dual luciferase assay (Figure 2D), thus confirming the functionality of the system. The luciferase assay was carried out using total protein extracts from the A947 strain grown in MA2 rich medium with $2 \%$ glucose as the carbon source for $40 \mathrm{~h}$. The activities of Renilla and firefly luciferases were measured as described in the Materials and Methods section. 


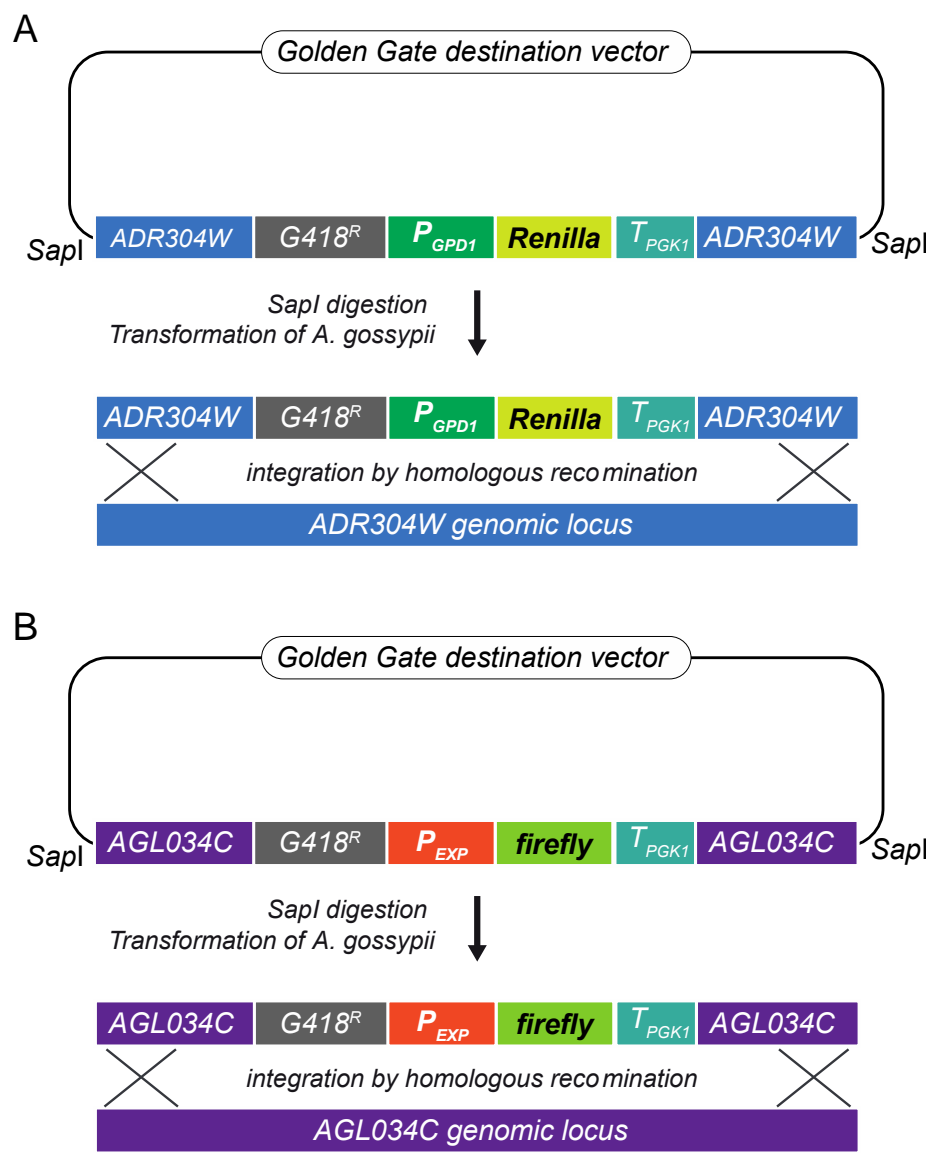

Figure 1. Assembly of the DLR (Dual Luciferase Reporter) system adapted for A. gossypii: (A) Integrative cassette for the expression of the Renilla luciferase under the control of the $P_{G P D 1}$ promoter; (B) Integrative cassette for the expression of the firefly luciferase under the control of the different experimental promoters. All the modules were assembled into a destination vector following a Golden Gate method. The integrative cassettes were obtained by SapI digestion and used for $A$. gossypii transformation. Genomic integration occurred by homologous recombination.

A

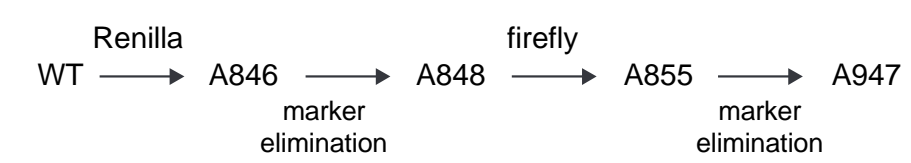

B
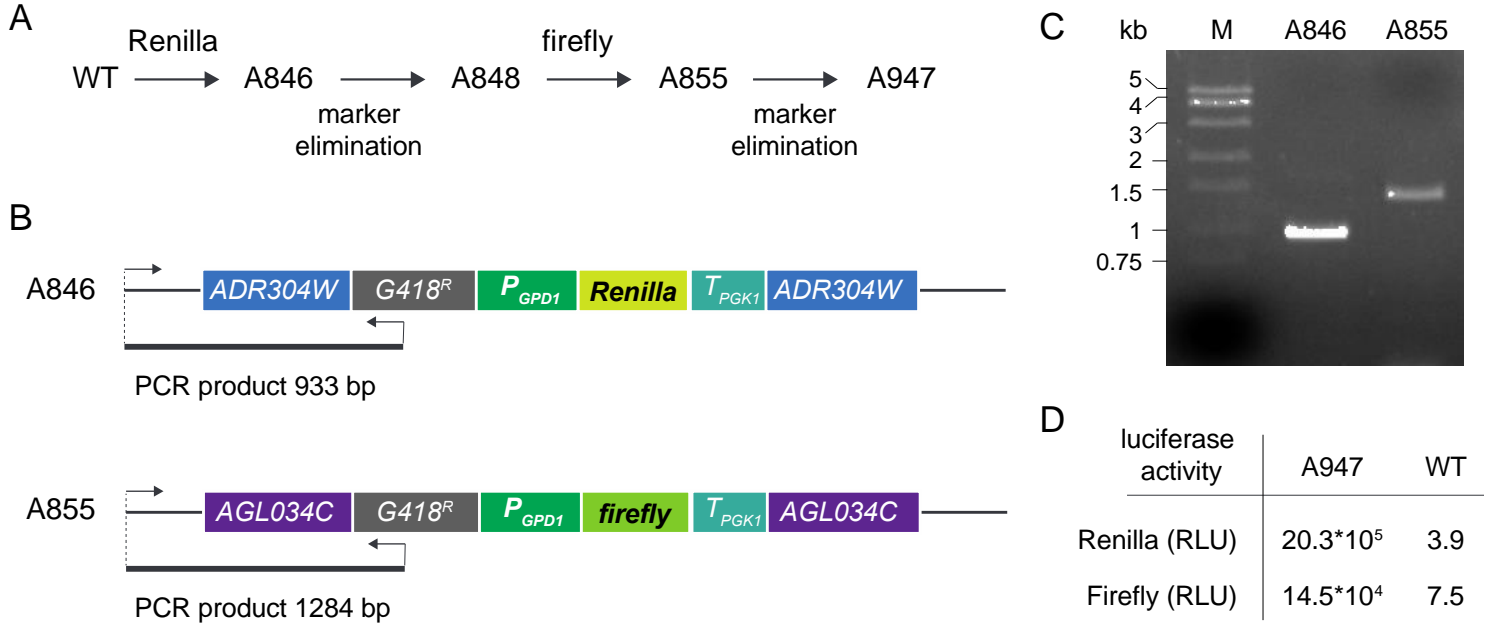

Figure 2. Construction of the DLR control strains: (A) Pipeline of the construction of the A947 control strain harboring both Renilla and firefly luciferases under the control of $P_{G P D 1}$; (B) Scheme for the ADR304W and AGL034C loci after genomic integration of the Renilla and firefly integrative cassettes; the strategy for the analytical PCRs is depicted; (C) Analytical PCR of the A. gossypii strains containing the Renilla (A846) and the firefly (A855) expression cassettes; (D) Renilla and firefly luciferase activities of the final A947 control strain and the WT expressed as Relative Light Units (RLU). 


\subsection{Dual luciferase Assay of Selected Promoter Sequences}

To validate the system for promoter analysis in A. gossypii, the intergenic sequences upstream the ATG codon from different genes of $A$. gossypii were challenged for their ability to drive the expression of the firefly luciferase. The promoter sequences were chosen according to our preliminary RNAseq data (unpublished results) and included a total of 10 genes that showed either higher, similar, or lower expression levels than the GPD1 (TDH3) gene (Table 1). The length of the selected promoter sequences extended from $176 \mathrm{bp}$ to $1000 \mathrm{bp}$, depending on each intergenic sequence (Table S4).

Table 1. Selected promoter sequences used in the present study.

\begin{tabular}{|c|c|c|c|c|c|}
\hline Gene & $\begin{array}{l}\text { Standard } \\
\text { Name }\end{array}$ & $\begin{array}{l}\text { S. cerevisiae } \\
\text { Homolog }\end{array}$ & $\begin{array}{l}\text { RNAseq } \\
\text { FKPM * }\end{array}$ & GO Description & $\begin{array}{l}\text { Promoter } \\
\text { Length (bp) }\end{array}$ \\
\hline AGR049W & CCW12 & YLR110C & 102847.2 & Cell wall mannoprotein & 432 \\
\hline AFR505C & TMA10 & $Y L R 327 C$ & 71752.9 & $\begin{array}{l}\text { Protein of unknown function that associates } \\
\text { with ribosomes }\end{array}$ & 258 \\
\hline$A C R 272 C$ & CWP1 & YKL096W & 28833.9 & $\begin{array}{l}\text { Cell wall mannoprotein that localizes to birth } \\
\text { scars of daughter cells }\end{array}$ & 950 \\
\hline AER312W & TSA1 & YML028W & 25764.9 & $\begin{array}{l}\text { Thioredoxin peroxidase. Panther family } \\
\text { PTHR10681 }\end{array}$ & 255 \\
\hline AER031C & $\begin{array}{c}G D P \\
(T D H 3)\end{array}$ & YGR192C & 15480.9 & $\begin{array}{l}\text { Glyceraldehyde-3-phosphate dehydrogenase. } \\
\text { Panther family PTHR10836 }\end{array}$ & 373 \\
\hline AGL366C & & No homolog & 14317.3 & & 672 \\
\hline AGR138W & SED1 & YDR077W & 13184.5 & $\begin{array}{l}\text { Major stress-induced structural GPI-cell wall } \\
\text { glycoprotein. Panther family PTHR35523 }\end{array}$ & 711 \\
\hline ADL036C & $C D A 2$ & YLR308W & 12871.6 & $\begin{array}{l}\text { Chitin deacetylase. Panther family } \\
\text { PTHR10587 }\end{array}$ & 325 \\
\hline AGR408W & HSP26 & YBR072W & 5158.0 & $\begin{array}{l}\text { Small heat shock protein (sHSP) with } \\
\text { chaperone activity }\end{array}$ & 1000 \\
\hline AFR038W & & YHR138C & 2681.4 & Protein of unknown function & 202 \\
\hline AFR132C & PFS1 & YHR185C & 72.8 & $\begin{array}{l}\text { Sporulation protein required for prospore } \\
\text { membrane formation }\end{array}$ & 176 \\
\hline
\end{tabular}

* FPKM, Fragments per kilobase per million mapped reads.

The promoter sequences were PCR amplified using specific primers (Table S3) that contained a BsaI recognition site and 4-nucleotide overhangs for the assembly of the integrative cassettes using a Golden Gate method. The control strain A848, containing the Renilla expression cassette (Figure 1A), was then transformed with the integrative cassettes containing the experimental reporter firefly luciferase under the control of different promoter sequences (Figure 1B). The integration of each expression cassette in the $A D R 304 \mathrm{~W}$ locus was confirmed by analytical PCR (not shown). Prior to the luciferase assay, the G418 ${ }^{\mathrm{R}}$ marker was eliminated in all the engineered strains (Table S1).

The activities of Renilla and firefly luciferases were measured in all the generated strains grown in MA2 rich medium with $2 \%$ glucose as the carbon source for $40 \mathrm{~h}$. The promoter activities of the analyzed sequences were compared with the activity of the strong promoter $P_{G P D 1}$. Our data showed that three sequences $\left(P_{S E D 1}, P_{C C W 12}\right.$, and $\left.P_{T S A 1}\right)$ provided a strong promoter activity, between 3 to 5 times higher than that of the $P_{G P D 1}$ (Figure $3 \mathrm{~A}$ ). In addition, the intergenic sequences corresponding to the genes HSP26, AGL366C, TMA10, CWP1, AFR038W, CDA2, and PFS1 were also able to drive the expression of the firefly luciferase, thereby confirming their promoter activity. These promoters showed a wide range of transcriptional activity from about 4 to 55 times lower than that of the $P_{G P D 1}$ (Figure $3 \mathrm{~B}$ ). Hence, the promoter sequences analyzed can be categorized into three classes according to their promoter activity: strong, medium, and weak promoters 
(Figure S2), offering a dynamic range of promoter activity. Remarkably, while the genes TMA10 and CWP1 exhibited higher transcription level than GPD1 (Table 1), their corresponding intergenic sequences showed very low promoter activity. Indeed, according to our results, the promoter activity of the intergenic sequences used in the analysis does not correlate directly with the transcription level of their corresponding genes (Figure S2), indicating that additional CIS-acting elements, either positive or negative, must be involved in the transcriptional regulation of the chosen genes. Consequently, the identification of new promoters for metabolic engineering cannot solely rely on gene expression analyses, but must be addressed by using efficient tools for promoter analysis, which can also be applied to uncover CIS-acting elements within a promoter sequence.
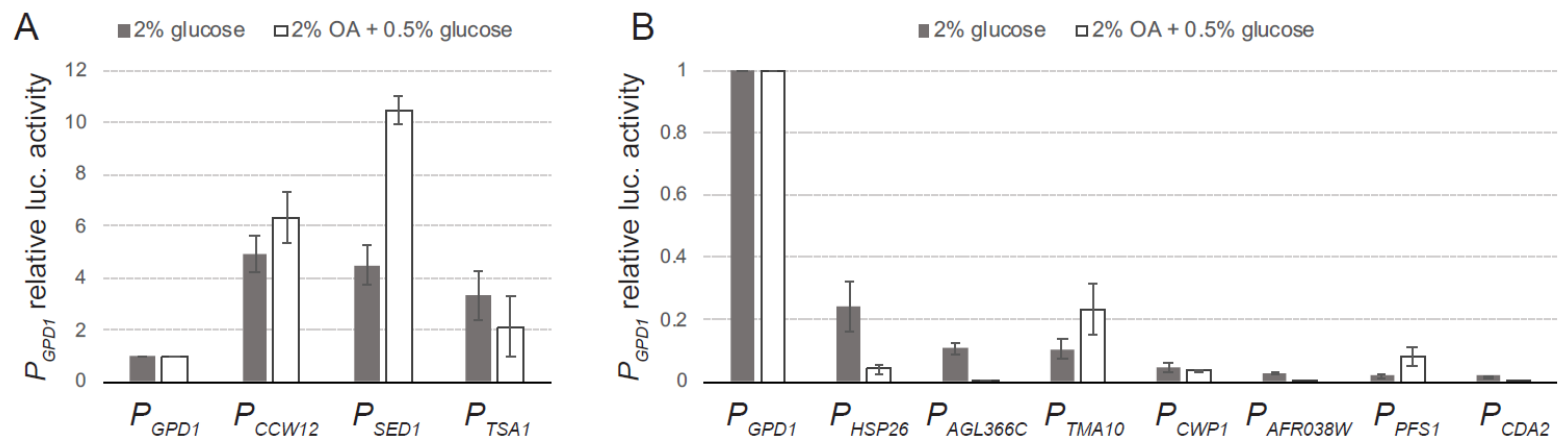

Figure 3. Luciferase assay of selected promoters: (A) DLR assay of strong promoters; (B) DLR assay of medium/weak promoters. The luciferase assays were carried out from cultures containing either $2 \%$ glucose (grey bars) or $2 \%$ oleic acid $(\mathrm{OA})+0.5 \%$ glucose (white bars). Data are the average of three independent experiments, performed in duplicate, and are expressed as relative luciferase activities with respect to $P_{G P D 1}$ activity.

The identification of inducible promoters with carbon source responsiveness also constitutes an important issue for metabolic engineering approaches. For example, the bioproduction of riboflavin using A. gossypii industrial strains employs low-cost oil as a carbon source [26], and, therefore, it is of interest to find regulatable promoters that can be either induced or repressed in oil-containing media. In this regard, we decided to evaluate the promoter activity of the selected sequences in cultures containing $2 \%$ oleic acid $(\mathrm{OA})$ plus $0.5 \%$ glucose. The addition of glucose promotes the rapid germination of the spores in the early stages of cultures, as described in [27]. Hence, the luciferase assay was performed from cultures grown for $40 \mathrm{~h}$, when the OA is essentially the only carbon source. Our data showed that several promoters exhibited substantial differences in their relative luciferase activity depending on the carbon source. While the promoter activity of $P_{S E D 1}, P_{T M A 10}$, and $P_{P F S 1}$ was significantly induced in OA-containing media, the activity of $P_{H S P 26}$ and $P_{A G L 366 C}$ was repressed compared with the cultures using glucose as the only carbon source (Figure 3). Thus, the promoter analysis used in this work represents an efficient tool for the identification of promoter sequences with different features, including regulatable abilities. Moreover, it is also possible to further characterize the regulatory properties of each promoter by a genetic dissection of the promoter CIS-acting elements. A. gossypii, which is mainly considered a microbial factory, also represents an adequate model organism in cell biology studies regarding polarized growth and ploidy $[28,29]$; therefore, the availability of new molecular tools will strongly benefit $A$. gossypii research.

\subsection{In Vivo Analysis of New Promoter Sequences}

Next, we wanted to confirm the results obtained from the luciferase assay in modifying the expression of an A. gossypii endogenous gene. The MSN2 gene was selected because the alteration on its expression level triggers major changes in the sporulation ability of $A$. gossypii [28]. We previously observed that the overexpression of MSN2 nearly abolished the sporulation ability of $A$. gossypii, whereas the $m s n 2 \Delta$ strain showed a significant increase in the sporulation capacity with respect to the wild-type strain (unpublished results). Hence, 
integrative expression cassettes including the promoter sequences of $P_{S E D 1}, P_{T S A 1}, P_{A G L 366 C}$, and $P_{A F R 038 W}$ were PCR-amplified using specific primers that provided recombinogenic flanks targeting the MSN2 gene of A. gossypii. Homokaryotic strains were obtained and the loxP-KanMX-loxP marker was eliminated in all the engineered strains. The expression level of the MSN2 gene was measured by qPCR in all the strains generated (Figure 4A). Our data showed that both $P_{S E D 1}$ and $P_{T S A 1}$ can be considered bona fide strong promoters that were able to provide a significantly higher expression level than the well-known $P_{G P D 1}$, which is generally used for gene overexpression. In addition, both $P_{A G L 366 C}$ and $P_{A F R 038 W}$ exhibited about 10 times less promoter strength than that of $P_{G P D 1}$, again confirming our previous results with the luciferase assay.

A

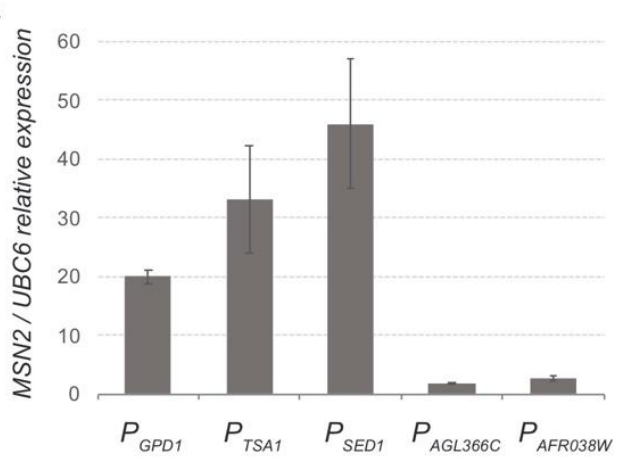

B

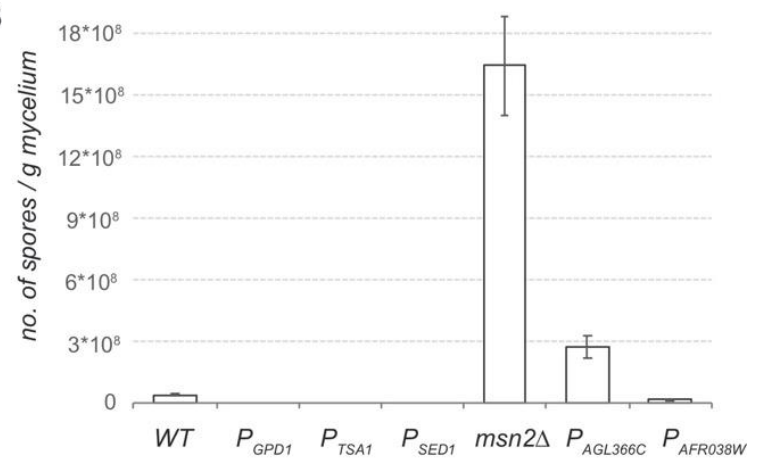

C
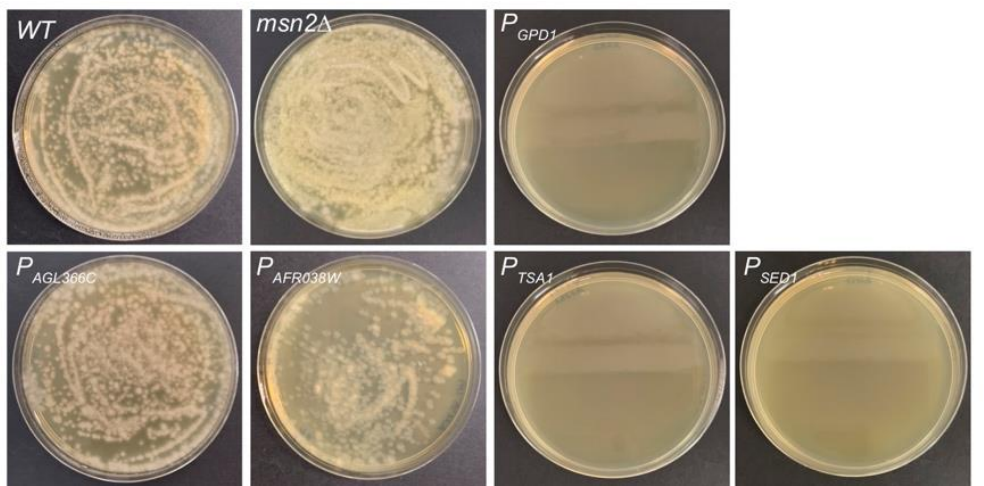

Figure 4. In vivo analysis of promoter sequences: (A) qPCR gene expression analysis of MSN2 controlled by different promoter sequences. Transcription levels of MSN2 were normalized using the A. gossypii UBC6 gene as a reference. The results are the means of two independent experiments performed in duplicate. (B) Sporulation analysis of A. gossypii strains where MSN2 is controlled by different promoter sequences. The wild-type and $m s n 2 \Delta$ strains are included as references. Spores were obtained from $100 \mathrm{mg}$ of mycelia from the different strains grown in SPA media. Data were obtained from three independent experiments. (C) Dilutions of the spore preparations were plated onto MA2 media for visualization.

As mentioned above, the expression level of MSN2 highly determines the sporulation ability of $A$. gossypii. Consequently, the sporulation rate was analyzed in the aforementioned strains, where the transcription of MSN2 is driven by different promoter sequences. Both the MSN2-overexpressing strain $\left(P_{G P D 1}\right)$ and the $m s n 2 \Delta$ strain were used as control strains. Our data showed that the utilization of strong promoters such as $P_{G P D 1}, P_{S E D 1}$, and $P_{T S A 1}$, which provided high levels of MSN2 expression, completely abolished the sporulation capacity of $A$. gossypii (Figure 4B,C). In contrast, the expression of MSN2 under the control of the weak promoters $P_{A G L 366 C}$ and $P_{A F R 038 W}$ maintained the sporulation ability within the range of the WT strain, but far below the sporulation rate of the $m s n 2 \Delta$ strain (Figure $4 B, C$ ).

Taken together, our results demonstrate a good correlation between the data obtained with the luciferase assays and the in vivo analysis. Hence, the promoters presented in this work will assist the implementation of multiple expression platforms for metabolic 
engineering in $A$. gossypii. Three new strong promoters $\left(P_{C C W 12}, P_{S E D 1}\right.$, and $\left.P_{T S A 1}\right)$ can be applied to gene overexpression platforms. Additionally, $P_{S E D 1}$ can be considered an effective option for gene overexpression in lipid-based culture media. In contrast, seven novel promoters $\left(P_{H S P 26}, P_{A G L 366 C}, P_{T M A 10}, P_{C W P 1}, P_{A F R 038 W}, P_{P F S 1}\right.$, and $\left.P_{C D A 2}\right)$ are available for gene underexpression, including lipid-dependent regulatory properties for $P_{H S P 26}$, $P_{A G L 366 C}, P_{T M A 10}$, and $P_{P F S 1}$. The adapted DLR system represents an efficient molecular tool for promoter analysis in A. gossypii beyond its biotechnological applications. For example, the OA-regulatable promoters can be used for the identification of oleate response elements (ORE), which participate in oleate induction [30]. In this regard, promoter tools have been described in other filamentous fungi such as Trichoderma reesei, Aspergillus spp., and Penicillium chrysogenum, which are also used for the production of industrially relevant metabolites [31-34], highlighting the importance of the identification of native promoters that can be used for the development of efficient expression platforms.

Supplementary Materials: The following are available online at https://www.mdpi.com/article/ 10.3390/jof7110906/s1: Figure S1: Map of the Golden Gate destination vector; Figure S2: Comparison between the gene expression level and the promoter activity of the selected sequences; Table S1: $A$. gossypii strains used in this study; Table S2: Relevant sequences of the integrative cassettes; Table S3: List of primers used in this study; Table S4: Intergenic sequences used for the promoter analyses.

Author Contributions: Conceptualization, J.L.R. and A.J.; Data curation, G.M.-F. and A.J.; Formal analysis, G.M.-F., J.-F.M.-B. and A.J.; Funding acquisition, J.L.R. and A.J.; Investigation, G.M.-F.; Methodology, G.M.-F., J.-F.M.-B., J.L.R. and A.J.; Project administration, J.L.R. and A.J.; Resources, G.M.-F. and A.J.; Supervision, A.J.; Validation, G.M.-F., J.L.R. and A.J.; Visualization, J.L.R. and A.J.; Writing—original draft, A.J.; Writing—review and editing, G.M.-F., J.-F.M.-B., J.L.R. and A.J. All authors have read and agreed to the published version of the manuscript.

Funding: This research was funded by the Spanish Ministerio de Economía y Competitividad, grant number BIO2017-88435-R, and Junta de Castilla y León, grant number SA016P17. GM-F was the recipient of an FPI predoctoral contract (PRE2018-084931) granted by MCIN/AEI/10.13039/ 501100011033. JFMB was the recipient of a postdoctoral contract (FJC2019-041035-I) granted by MCIN/AEI/10.13039/501100011033.

Institutional Review Board Statement: Not applicable.

Informed Consent Statement: Not applicable.

Data Availability Statement: Data is contained within the article or supplementary material.

Acknowledgments: We thank María Dolores Sánchez and Silvia Domínguez for excellent technical help.

Conflicts of Interest: The authors declare no conflict of interest.

\section{References}

1. Aguiar, T.Q.; Silva, R.; Domingues, L. New biotechnological applications for Ashbya gossypii: Challenges and perspectives. Bioengineered 2016, 8, 309-315. [CrossRef]

2. Revuelta, J.L.; Ledesma-Amaro, R.; Lozano-Martinez, P.; Díaz-Fernández, D.; Martinez-Buey, R.; Jiménez, A. Bioproduction of riboflavin: A bright yellow history. J. Ind. Microbiol. Biotechnol. 2017, 44, 659-665. [CrossRef]

3. Serrano-Amatriain, C.; Ledesma-Amaro, R.; López-Nicolás, R.; Ros, G.; Jiménez, A.; Revuelta, J.L. Folic Acid Production by Engineered Ashbya gossypii. Metab. Eng. 2016, 38, 473-482. [CrossRef]

4. Ledesma-Amaro, R.; Buey, R.M.; Revuelta, J.L. Increased production of inosine and guanosine by means of metabolic engineering of the purine pathway in Ashbya gossypii. Microb. Cell Factories 2015, 14, 1-8. [CrossRef]

5. Ledesma-Amaro, R.; Lozano-Martínez, P.; Jiménez, A.; Revuelta, J.L. EngineeringAshbya gossypiifor efficient biolipid production. Bioengineered 2015, 6, 119-123. [CrossRef]

6. Aguiar, T.Q.; Silva, R.; Domingues, L. Ashbya gossypii beyond industrial riboflavin production: A historical perspective and emerging biotechnological applications. Biotechnol. Adv. 2015, 33, 1774-1786. [CrossRef] [PubMed]

7. Silva, R.; Aguiar, T.Q.; Coelho, E.; Jiménez, A.; Revuelta, J.L.; Domingues, L. Metabolic engineering of Ashbya gossypii for deciphering the de novo biosynthesis of $\gamma$-lactones. Microb. Cell Factories 2019, 18, 62. [CrossRef] [PubMed]

8. Díaz-Fernández, D.; Aguiar, T.Q.; Martín, V.I.; Romaní, A.; Silva, R.; Domingues, L.; Revuelta, J.L.; Jiménez, A. Microbial lipids from industrial wastes using xylose-utilizing Ashbya gossypii strains. Bioresour. Technol. 2019, 293, 122054. [CrossRef] [PubMed] 
9. Lozano-Martínez, P.; Martinez-Buey, R.; Ledesma-Amaro, R.; Jiménez, A.; Revuelta, J.L. Engineering Ashbya gossypii strains for de novo lipid production using industrial by-products. Microb. Biotechnol. 2016, 10, 425-433. [CrossRef]

10. Schwechheimer, S.K.; Park, E.Y.; Revuelta, J.L.; Becker, J.; Wittmann, C. Biotechnology of riboflavin. Appl. Microbiol. Biotechnol. 2016, 100, 2107-2119. [CrossRef]

11. Jiménez, A.; Muñoz-Fernández, G.; Ledesma-Amaro, R.; Martinez-Buey, R.; Revuelta, J.L. One-vector CRISPR/Cas9 genome engineering of the industrial fungus Ashbya gossypii. Microb. Biotechnol. 2019, 12, 1293-1301. [CrossRef] [PubMed]

12. Jiménez, A.; Hoff, B.; Revuelta, J.L. Multiplex genome editing in Ashbya gossypii using CRISPR-Cpf1. New Biotechnol. 2020, 57, 29-33. [CrossRef] [PubMed]

13. Ledesma-Amaro, R.; Jiménez, A.; Revuelta, J.L. Pathway Grafting for Polyunsaturated Fatty Acids Production in Ashbya gossypii through Golden Gate Rapid Assembly. ACS Synth. Biol. 2018, 7, 2340-2347. [CrossRef] [PubMed]

14. Ro, D.-K.; Paradise, E.M.; Ouellet, M.; Fisher, K.J.; Newman, K.L.; Ndungu, J.M.; Ho, K.A.; Eachus, R.A.; Ham, T.S.; Kirby, J.; et al. Production of the antimalarial drug precursor artemisinic acid in engineered yeast. Nat. Cell Biol. 2006, 440, 940-943. [CrossRef] [PubMed]

15. Xue, Z.; Sharpe, P.L.; Hong, S.-P.; Yadav, N.S.; Xie, D.; Short, D.R.; Damude, H.G.; Rupert, R.A.; Seip, J.E.; Wang, J.; et al. Production of omega-3 eicosapentaenoic acid by metabolic engineering of Yarrowia lipolytica. Nat. Biotechnol. 2013, 31, 734-740. [CrossRef]

16. Gassler, T.; Sauer, M.; Gasser, B.; Egermeier, M.; Troyer, C.; Causon, T.; Hann, S.; Mattanovich, D.; Steiger, M.G. The industrial yeast Pichia pastoris is converted from a heterotroph into an autotroph capable of growth on $\mathrm{CO}_{2}$. Nat. Biotechnol. 2020, 38, 210-216. [CrossRef]

17. Ledesma-Amaro, R.; Serrano-Amatriain, C.; Jiménez, A.; Revuelta, J.L. Metabolic engineering of riboflavin production in Ashbya gossypii through pathway optimization. Microb. Cell Factories 2015, 14, 1-8. [CrossRef] [PubMed]

18. Jiménez, A.; Santos, M.A.; Revuelta, J.L. Phosphoribosyl pyrophosphate synthetase activity affects growth and riboflavin production in Ashbya gossypii. BMC Biotechnol. 2008, 8, 67. [CrossRef]

19. Dünkler, A.; Wendland, J. Use of MET3 promoters for regulated gene expression in Ashbya gossypii. Curr. Genet. 2007, 52, 1-10. [CrossRef]

20. Kaufmann, A. A plasmid collection for PCR-based gene targeting in the filamentous ascomycete Ashbya gossypii. Fungal Genet. Biol. 2009, 46, 595-603. [CrossRef]

21. Jiménez, A.; Santos, M.A.; Pompejus, M.; Revuelta, J.L. Metabolic Engineering of the Purine Pathway for Riboflavin Production in Ashbya gossypii. Appl. Environ. Microbiol. 2005, 71, 5743-5751. [CrossRef]

22. Aguiar, T.Q.; Dinis, C.; Domingues, L. Cre-loxP-based system for removal and reuse of selection markers in Ashbya gossypii targeted engineering. Fungal Genet. Biol. 2014, 68, 1-8. [CrossRef]

23. Mateos, L.; Jiménez, A.; Revuelta, J.L.; Santos, M.A. Purine Biosynthesis, Riboflavin Production, and Trophic-Phase Span Are Controlled by a Myb-Related Transcription Factor in the Fungus Ashbya gossypii. Appl. Environ. Microbiol. 2006, 72, 5052-5060. [CrossRef] [PubMed]

24. Livak, K.J.; Schmittgen, T.D. Analysis of relative gene expression data using real-time quantitative PCR and the 2- $\Delta \Delta C T$ method. Methods 2001, 25, 402-408. [CrossRef]

25. Ledesma-Amaro, R.; Santos, M.A.; Jiménez, A.; Revuelta, J.L. Strain Design of Ashbya gossypii for Single-Cell Oil Production. Appl. Environ. Microbiol. 2014, 80, 1237-1244. [CrossRef] [PubMed]

26. Stahmann, K.-P.; Revuelta, J.L.; Seulberger, H. Three biotechnical processes using Ashbya gossypii, Candida famata, or Bacillus subtilis compete with chemical riboflavin production. Appl. Microbiol. Biotechnol. 2000, 53, 509-516. [CrossRef]

27. Díaz-Fernández, D.; Lozano-Martínez, P.; Buey, R.M.; Revuelta, J.L.; Jiménez, A. Utilization of xylose by engineered strains of Ashbya gossypii for the production of microbial oils. Biotechnol. Biofuels 2017, 10,1-12. [CrossRef]

28. Wendland, J. Sporulation in Ashbya gossypii. J. Fungi 2020, 6, 157. [CrossRef]

29. Anderson, C.A.; Roberts, S.; Zhang, H.; Kelly, C.M.; Kendall, A.; Lee, C.; Gerstenberger, J.; Koenig, A.B.; Kabeche, R.; Gladfelter, A.S. Ploidy variation in multinucleate cells changes under stress. Mol. Biol. Cell 2015, 26, 1129-1140. [CrossRef] [PubMed]

30. Gurvitz, A.; Rottensteiner, H. The biochemistry of oleate induction: Transcriptional upregulation and peroxisome proliferation. Biochim. Biophys. Acta (BBA)-Bioenerg. 2006, 1763, 1392-1402. [CrossRef]

31. Fleißner, A.; Dersch, P.; Fleissner, A. Expression and export: Recombinant protein production systems for Aspergillus. Appl. Microbiol. Biotechnol. 2010, 87, 1255-1270. [CrossRef] [PubMed]

32. Polli, F.; Meijrink, B.; Bovenberg, R.A.; Driessen, A.J. New promoters for strain engineering of Penicillium chrysogenum. Fungal Genet. Biol. 2016, 89, 62-71. [CrossRef] [PubMed]

33. Fitz, E.; Wanka, F.; Seiboth, B. The Promoter Toolbox for Recombinant Gene Expression in Trichoderma reesei. Front. Bioeng. Biotechnol. 2018, 6, 135. [CrossRef]

34. Umemura, M.; Kuriiwa, K.; Dao, L.V.; Okuda, T.; Terai, G. Promoter tools for further development of Aspergillus oryzae as a platform for fungal secondary metabolite production. Fungal Biol. Biotechnol. 2020, 7, 1-9. [CrossRef] [PubMed] 